\title{
Association of aortic coarctation and malignant right coronary artery anomaly: an unusual cause of angina pectoris
}

This article was published in the following Dove Press journal:

International Medical Case Reports Journal

I 3 July 201 I

Number of times this article has been viewed

\author{
Rubens Sirtoli Filho' \\ Leonardo Cao Cãmbra \\ de Almeida' \\ Maysa Godoy Gomes \\ Mazurek Sirtoli' \\ Liliana Pena Pilatti ${ }^{2}$ \\ Marcelo Valladão de \\ Carvalho ${ }^{2}$ \\ Marcelo Schafranski ${ }^{3}$ \\ 'Real e Benemérita Associação \\ Portuguesa de Beneficência, \\ São Paulo, Brazil; ${ }^{2}$ Department of \\ Hemodynamics, ${ }^{3}$ Department of \\ Intensive Care, Hospital Bom Jesus, \\ Ponta Grossa, Brazil
}

\begin{abstract}
A 53-year-old man with exercise-induced ischemia was referred for investigation. Coronary angiography revealed a right coronary artery originating from the trunk of the left coronary artery, and an aortic coarctation was observed on aortography. A CT angiogram confirmed these findings. Resection of the aortic coarctation and reimplantation of the ostial portion of right coronary artery into its native site was performed, and resulted in a satisfactory outcome. The association of an anomalous right coronary artery with aortic coarctation has rarely been described and represents a critical situation where early diagnosis and prompt intervention are essential.
\end{abstract}

Keywords: coronary anomalies, single ostium, myocardial ischemia, angina pectoris

\section{Introduction}

A single coronary ostium is an entity uncommonly observed, accounting for only about $24 \%$ of coronary anomalies. ${ }^{1}$ Its prevalence on the general population is around $1.3 \%$, and represents quite a rare finding in cineangiocoronariographic exams, ${ }^{1}$ with incidence rates ranging from $0.0024 \%$ to $0.066 \%$. The vast majority of cases are discovered accidentally on routine investigation or on risk stratification tests requested for patients with suspected coronary artery disease. ${ }^{2}$ These variations may be associated with other cardiac defects, such as valvulopathies and congenital heart diseases (tetralogy of Fallot, aortic coarctation, and transposition of the great vessels). ${ }^{3}$ A single coronary artery is amongst the rarest anomalies ( $8.8 \%$ ), and deserves special attention because any kind of alteration that predisposes to a reduced blood flow in this vessel can ultimately result in more extensive myocardial damage. ${ }^{4}$ Definitive diagnosis can be confirmed by angiography and computed tomography (CT) angiography, the latter being responsible for the identification of the anatomic coronary course related to the great vessels of the heart. The interarterial course, defined as the passage of the coronary artery between the aorta and the pulmonary artery, is associated with sudden death during or after exertion, mainly in young patients. ${ }^{5}$ Here we report the rare association of a right coronary artery anomaly, exercise-induced ischemia, and aortic coarctation, in a scenario where early diagnosis was made and the appropriate surgical procedure resulted in a successful outcome.

\section{Case report}

A 53-year-old man with a history of hypertension, dyslipidemia, and stable angina CCS 2 underwent an exercise tolerance test which was positive for stress-induced ischemia (Figure 1A and 1B). Based on this result, the patient was referred to our 
Serviço de Ergometria

R. REAL E BENEMÉRITA SOCIEDADE ECG REPOUSO E M PE

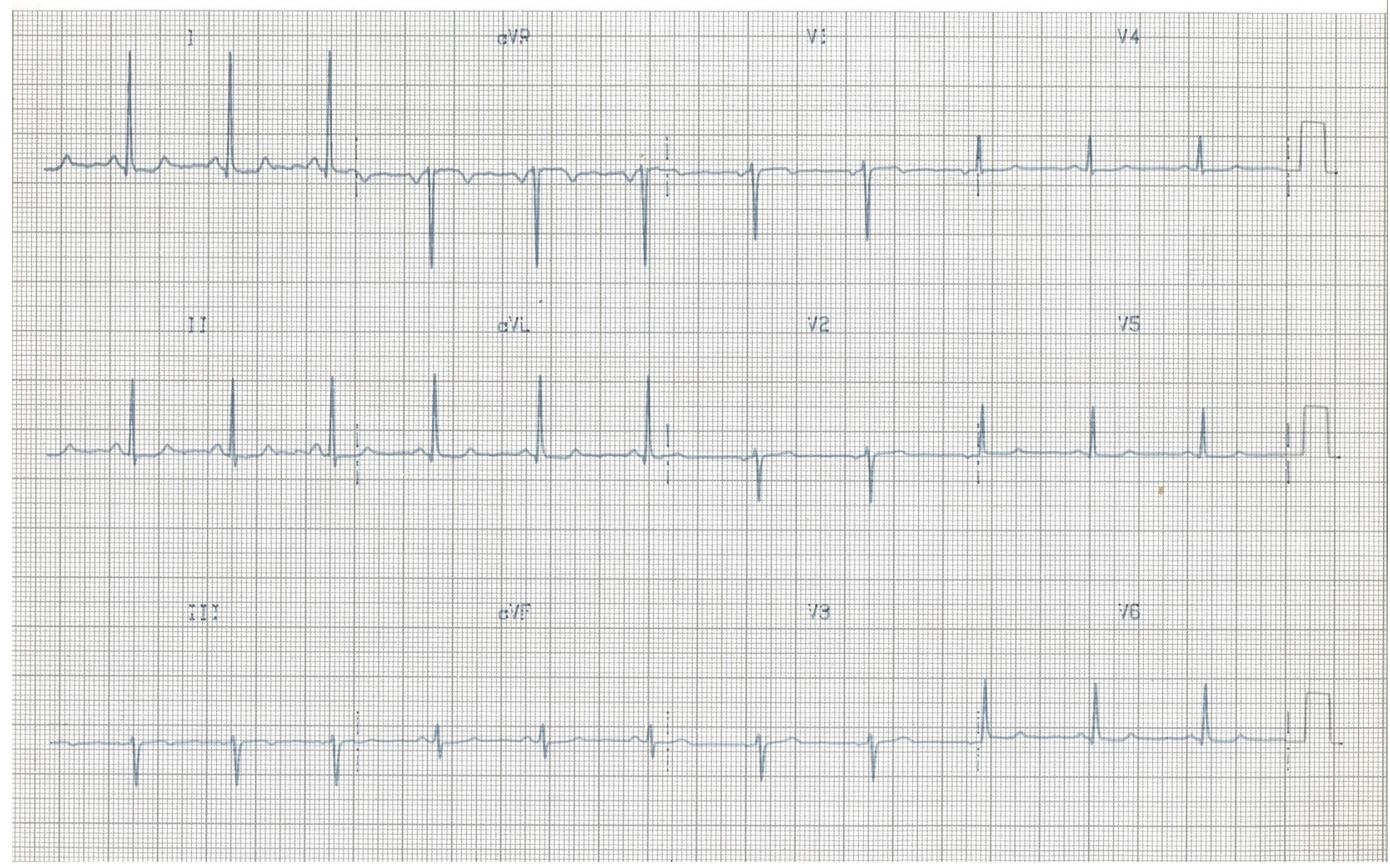

Figure IA Resting electrocardiogram showing no abnormalities.

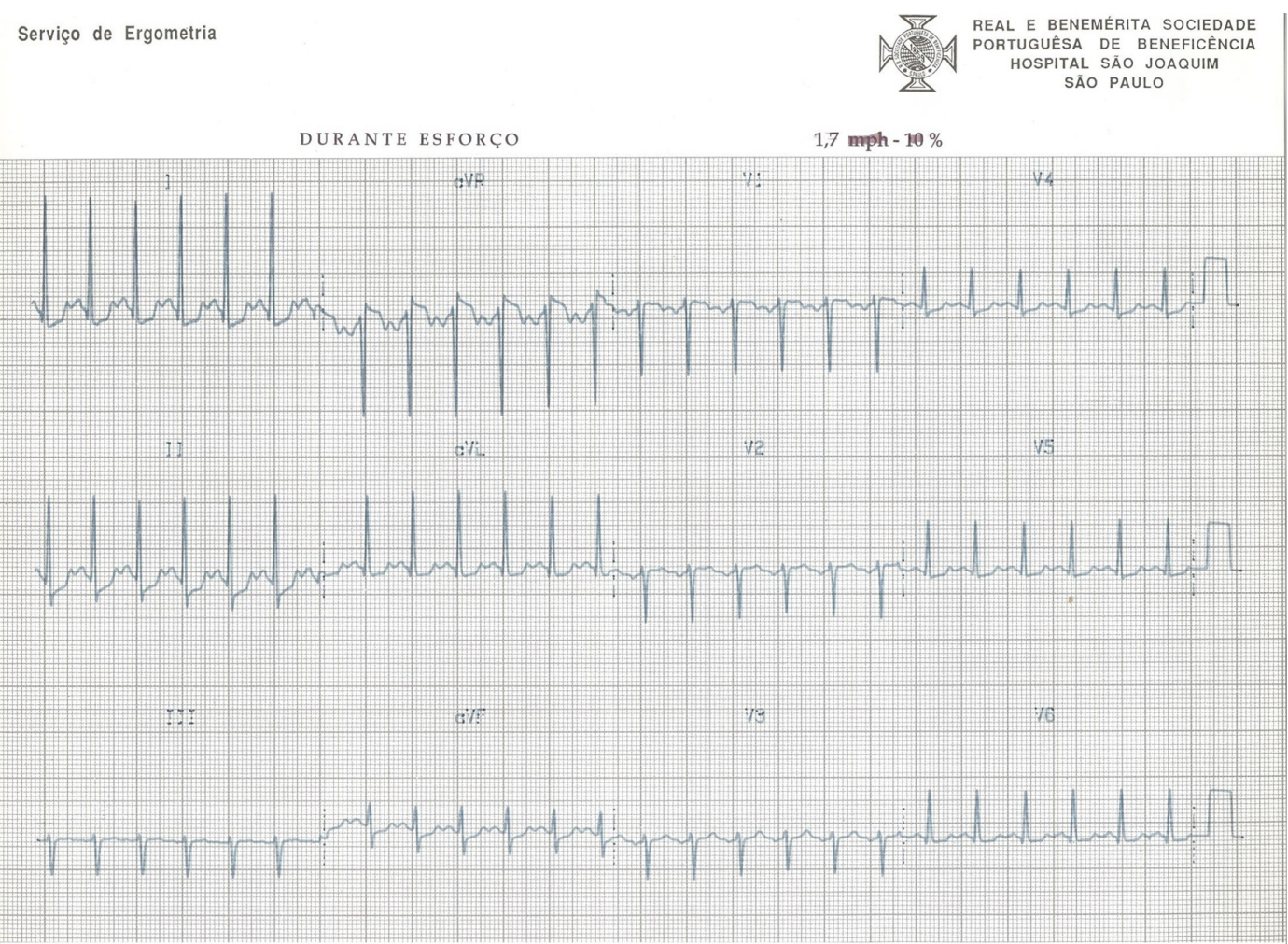

Figure IB Electrocardiogram showing ST segment depression mainly in leads I, II, aVL, and aVF. 
service for further investigation. Physical examination was unremarkable, with no dyspnea, lung congestion, signs of increased venous pressure, gallops, murmurs, or rubs. His heart rate was 87 beats per minute and blood pressure was stable at $110 / 70 \mathrm{mmHg}$. The ankle-brachial index in his right leg was 0.76 . His daily medications included aspirin $200 \mathrm{mg}$, atenolol $50 \mathrm{mg}$, and atorvastatin $20 \mathrm{mg}$. An electrocardiogram on admission demonstrated sinus rhythm, with a PR interval of 0.12 seconds, a QRS interval of 0.10 seconds, $\mathrm{AQRS}+30$, with no repolarization abnormalities or evidence of injury. The chest X-ray showed widening of the aortic shadow. Laboratory investigations showed a normal full blood count, and normal liver and kidney tests. The patient underwent coronary angiography through the right femoral artery, which revealed aortic coarctation (Figure 2) and a right dominance pattern, with an abnormal right coronary origin, arising from the left coronary artery branch, with no obstructive lesions (Figure 3). No extrinsic compression was observed and the remaining coronary arteries showed no evidence of relevant atherosclerotic obstruction. Left ventricular function was preserved, with no segmental hypokinesia. Although coronary angiography did not show any significant obstructive lesions, a CT coronary angiogram (using a clinical scanner with 64 data channels) was performed, which revealed an anomalous right coronary course, passing between the aorta and the exit tract of the right ventricle (interarterial, Figure 4), and confirmed the aortic coarctation (Figure 5). A transthoracic echocardiogram showed no further malformations. Based on the positive findings of the exercise test and imaging studies which revealed the malignant course of the right coronary artery, as well as the aortic coarctation, surgical correction of the aortic aneurysm (resection with end-to-end anastomosis) and reimplantation of the coronary ostium were indicated. The surgical procedure was performed successfully with no complications. The patient was discharged from hospital on the fifth postoperative day taking atorvastatin $20 \mathrm{mg}$ /day and atenolol $25 \mathrm{mg}$ twice daily. Three months after the

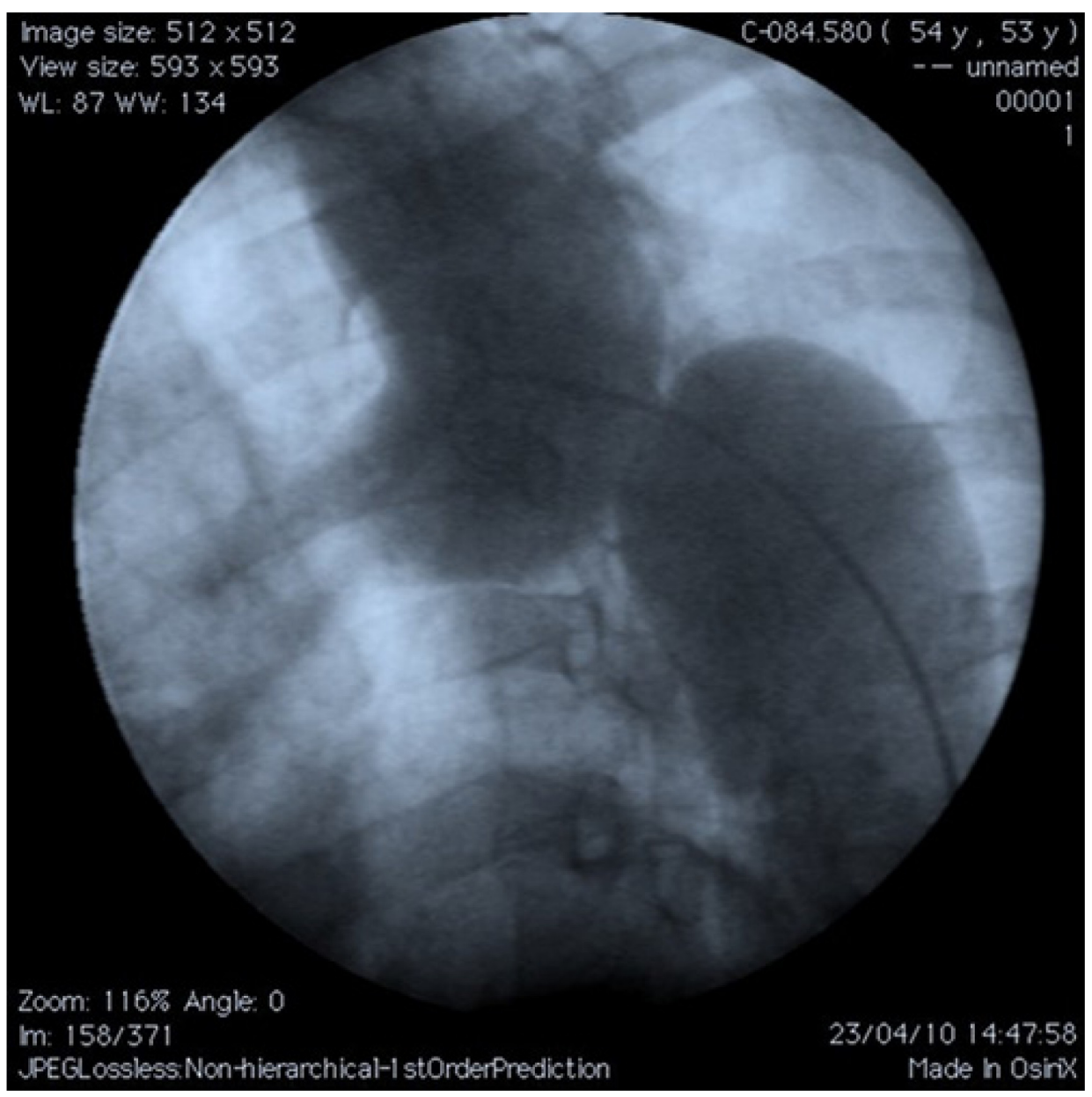

Figure 2 Angiogram demonstrating aortic coarctation. 


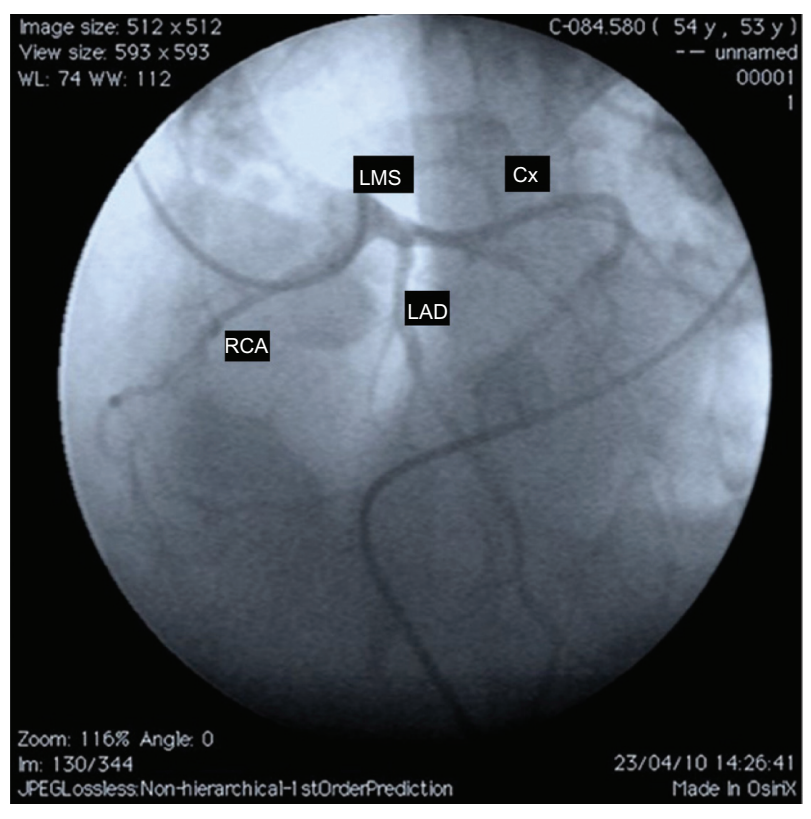

Figure 3 Coronary angiography revealing a single coronary ostium. The right coronary arises from the left coronary main branch.

Abbreviations: LMS, left main stem; Cx, circumflex artery; RCA, right coronary artery; LAD, left anterior descending artery.

procedure, the patient resumed his normal daily activities with no signs or symptoms of myocardial ischemia.

\section{Discussion}

The single coronary ostium is a clinical entity that runs a benign and asymptomatic clinical course in $85 \%$ of

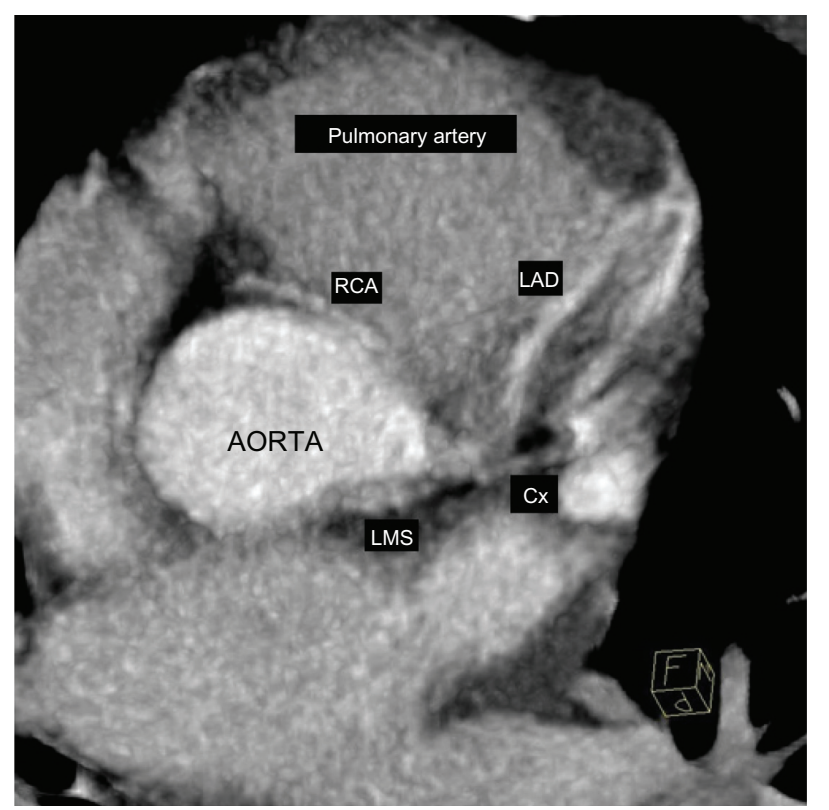

Figure $4 \mathrm{CT}$ angiogram showing the right coronary course between the aorta and pulmonary artery.

Abbreviations: LMS, left main stem; Cx, circumflex artery; RCA, right coronary artery; LAD, left anterior descending artery; RC, right coronary.

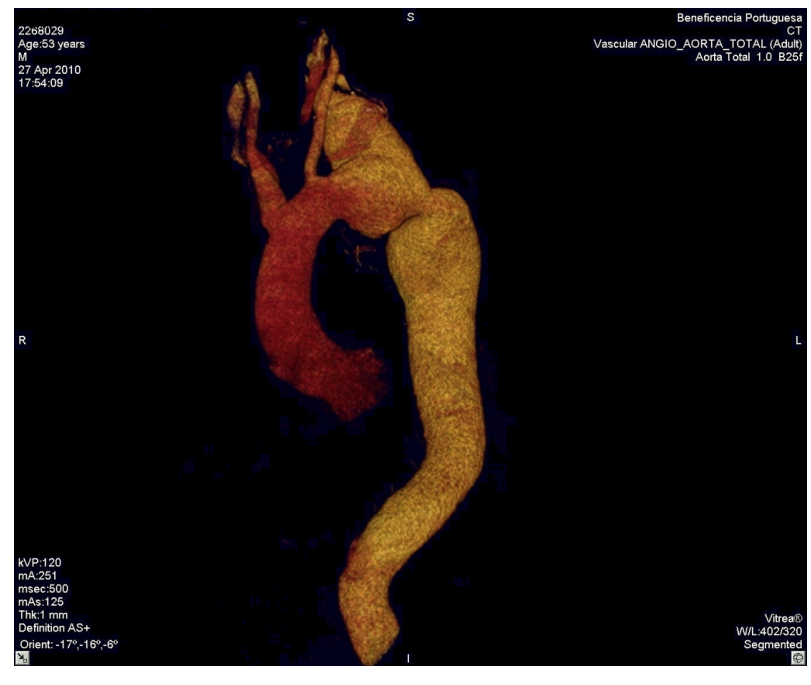

Figure $5 \mathrm{CT}$ angiography (three-dimensional reconstruction) confirming the aortic isthmus coarctation.

cases. ${ }^{6}$ However, around $15 \%$ of individuals may develop myocardial ischemia of varying degrees as a consequence of anomalies of the right coronary artery, which is most commonly diagnosed incidentally on angiography requested to investigate a range of and sometimes nonspecific chest complaints. ${ }^{2}$ These anomalies are divided into two major groups, according to whether or not they result in exerciseinduced ischemia as a consequence of extrinsic compression arising from their anomalous course between the aorta and the outflow tract of the right ventricle. Reduced blood flow through a compressed artery can result in myocardial ischemia and its clinical manifestations, which range from typical angina to malignant and fatal arrhythmias, sometimes followed by sudden death. ${ }^{7}$ The association of coronary anomalies with aortic coarctation is a very rare finding and both left ${ }^{8}$ and right ${ }^{9}$ coronary anomalies have been described in association with this great vessel malformation. Anatomopathological studies suggest that in individuals who present cardiac ischemia as a result of the anomalous course of the right coronary artery (arising from the contralateral sinus), restriction of blood flow occurs in rare and isolated episodes. The consequential accumulative ischemic damage leads to islands of necrotic tissue and myocardial fibrosis, paving grounds to electrical instability and potentially lethal high-rate arrhythmias. ${ }^{5}$ Possible ischemia-related factors are the ostium slit-shape formed by the origin of the anomalous vessel, which can be compressed during ventricular systole, as well as compression of the anomalous vessel that runs through the aorta and the pulmonary artery, especially during physical exertion, when these two major vessels dilate. 
The role of extrinsic compression by these major vessels is still elusive, as long as the hydrostatic pressure within the pulmonary artery is lower than the coronary perfusion pressure. ${ }^{2}$ Studies with intracoronary ultrasound were quite consistent in demonstrating that in this kind of anomaly. The role of the extrinsic compression by these major vessels is still a matter of debate. Although the hydrostatic pressure within the pulmonary artery is lower than the coronary perfusion pressure, ${ }^{2}$ studies with intracoronary ultrasound were quite consistent in demonstrating that in this kind of anomaly, the ectopic coronary artery follows, right after its origin, a transmural course, which is the site of greatest proximity between the aorta and the pulmonary artery. In this intramural segment, the external and internal coronary walls are thin and delicate, predisposing to compression of the vessel lumen in pace with the cardiac cycle, with phasic exacerbation during ventricular systole. Individual variances with respect to systolic compression may explain the different clinical and prognostic behaviors observed amongst subjects presenting with this condition. In this intramural segment, the external and internal aortic walls are thin and delicate, predisposing to compression of the vessel lumen in pace with the cardiac cycle, with phasic exacerbation during ventricular systole. Individual variances with respect to systolic compression may explain the different clinical and prognostic patterns observed in subjects presenting with this condition.

In most affected patients, the anomaly follows a benign course, without any major clinical repercussions. Even amongst the sudden death survivors, about 55\%-93\% describe no evidence of previous signs or symptoms consistent with the disease. About $10 \%$ of subjects present with variable degrees of dyspnea, palpitations, dizziness, syncope, and angina. Sudden death typically occurs in young patients during or after episodes of intense physical activity. The remaining symptoms are often observed in young adults and are associated with the development of systemic arterial hypertension, suggesting that stiffness of the aortic wall may represent a protective factor against sudden death in this subgroup of patients. ${ }^{10}$

Once the diagnosis of the anomaly is established, patients should avoid intense and competitive physical activities, and pharmacological therapy that includes a beta-blocker should be initiated promptly. ${ }^{2,11}$ Surgical procedures may also be indicated for this type of anomaly. Conventional revascularization, ostium reimplantation on the aortic root, excision of the common wall between the aorta and the anomalous vessel, and the creation of a new ostium at the end of the intramural segment of the vessel are all plausible alternatives. Martins et al describe a patient with an anomalous origin of the right coronary who underwent myocardial revascularization with right internal thoracic artery grafting to the proximal right coronary artery segment and proximal ligation of the right coronary artery, with a good postoperative outcome. ${ }^{10}$ Repair surgery can be obtained by right coronary artery reimplantation, or by using a graft for this coronary. ${ }^{12}$ It is necessary to perform a re-evaluation of the treated vessel before the patient is considered able to resume his normal physical activities. ${ }^{2,11}$

\section{Conclusion}

Technological advances in investigative imaging procedures have made the diagnosis of coronary anomalies quite accurate. Once a single ostium-type coronary anomaly is detected, it is mandatory to undertake CT angiographic evaluation of the coronary course. This measurement allows correct identification of patients in the subgroup of potentially malignant interarterial anatomy, who have the worst prognosis. The association of an anomalous right coronary artery with aortic coarctation has rarely been described and is a critical situation where early diagnosis and prompt intervention are essential to avoid serious and even fatal complications.

\section{Disclosure}

The authors report no conflicts of interest in this work.

\section{References}

1. Earls JP. Coronary artery anomalies. Tech Vasc Interv Radiol. 2006;9: 210-217.

2. Akcay A, Tuncer C, Batyraliev T. Isolated single coronary artery: a series of 10 cases. Circ J. 2008;72:1254-1258.

3. Harikrishnan S, Jacob SP, Tharakan J. Congenital coronary anomalies of origin and distribution in adults: a coronary arteriographic study. Indian Heart J. 2002;54:271-275.

4. Basso C, Maron BJ, Corrado D, Thiene G. Clinical profile of congenital coronary artery anomalies with origin from the wrong aortic sinus leading to sudden death in young competitive athletes. J Am Coll Cardiol. 2000;35:1493-1501.

5. Maron BJ. Sudden death in young athletes. $N$ Engl J Med. 2003;349:1064-1075.

6. Yamanaka O, Hobbs RE. Coronary artery anomalies in 126,595 patients undergoing coronary arteriography. Cathet Cardiovasc Diagn. 1990;21:28-40.

7. Kang JW, Seo JB, Chae EJ. Coronary artery anomalies: classification and electrocardiogram-gated multidetector computed tomographic findings. Semin Ultrasound CT MR. 2008;29:182-194.

8. Khanna A. Anomalous origin of the left coronary artery from the pulmonary artery in adulthood on CT and MRI. AJR Am J Roentgenol. 2005;185:326-329.

9. Rius T, Goyenechea M, Poon M. Combined cardiac congenital anomalies assessed by multi-slice spiral computed tomography. Eur Heart J. 2006;27:637. 
10. Martins MS, Bastos BS, Annibal JB, Bezerra AB. Myocardial revascularization in anomalous origin of the right coronary artery: case report. Rev Bras Cir Cardiovasc. 2007;22:505-508.

11. Angelini P. Coronary artery anomalies: an entity in search of an identity. Circulation. 2007;115:1296-1305.
12. Di Lello F, Mnuk JF, Flemma RJ, Mullen DC. Successful coronary reimplantation for anomalous origin of the right coronary artery from the left sinus of Valsalva. J Thorac Cardiovasc Surg. 1991;102:455-456.

\section{Publish your work in this journal}

The International Medical Case Reports Journal is an international, peer-reviewed open-access journal publishing original case reports from all medical specialties. Previously unpublished medical posters are also accepted relating to any area of clinical or preclinical science. Submissions should not normally exceed 2,000 words or
4 published pages including figures, diagrams and references. The manuscript management system is completely online and includes a very quick and fair peer-review system, which is all easy to use. Visit http://www.dovepress.com/testimonials.php to read real quotes from published authors.

Submit your manuscript here: http://www.dovepress.com/international-medical-case-reports-journal-journal 